МРТ В АИАГНОСТИКЕ РЕАКОГО САУЧАЯ ПЕРВИЧНО-МНОЖЕСТВЕННЫХ ЗАОКАЧЕСТВЕННЫХ ОБРАЗОВАНИЙ: ОСТЕОСАРКОМА И УРОТЕАИААЬНЫЙ РАК МОЧЕВОГО ПУЗЫРЯ, ААЕНОКАРЦИНОМА ПРЕАСТАТЕАЬНОЙ ЖЕАЕЗЫ

\author{
Григорьев Е.Г., Фролова И.Г., Перельмутер В.М., С^онимская Е.М., \\ ГольАберг В.Е., Фесик Е.А., Усынин Е.А., ГольАберг А.В.
}

$\Pi$

редставить наблюдение редкого сочетания опухолевой патологии мочевого пузыря, демонстрирующее возможности магнитно-резонансной томографии (MPT) в диагностике опухолей органов малого таза.

Материалы и методы. Исследование пациента Г., 68 мет, обратившегося с жалобами на наличие крови в моче, боли в животе и подвздошной области. Была заподозрена опухоль мочевого пузыря. Выполнены исследования: МРТ органов малого таза с целью уточнения мокализации образования, степени инвазии и планирования дальнейшего мечения.

Результаты. Диагноз опухоли мочевого пузыря подтвержден, выявлены образования разной гистологической структуры, симулирующие инвазию мышечного слоя мочевого пузыря.

Заключение. Представленный случай подтверждает ключевую роль МРТ в диагностике патологии органов малого таза, в частности - опухолей мочевого пузыря, с возможностью оценки распространенности патологического процесса и планировании цечения. Однако, учитывая многообразие возможных гистологических вариантов, для окончательного диагноза необходимо патоморфологическое подтверждение.

Ключевые слова: МРТ, динамическое контрастирование, диффузионновзвешенные изображения, опухоць мочевого пузыря, саркома.

Контактный автор: Григорьев Е.Г, e-mail: mrtomo@mail.ru

Для иитирования: Григорьев Е.Г., Фролова И.Г., Перельмутер В.М., Слонимская Е.М., Гольдберг В.Е., Фесик Е.А., Усынин Е.А., Гольдберг А.В. МРТ в диагностике редкого случая первично-множественных злокачественных образований: остеосаркома и уротелиальный рак мочевого пузыря, аденокариинома предстательной железы. REJR 2019; 9(2):288-294. DOI:10.21569/2222-7415-2019-9-2-288-294.
Статья получена:
27.04.19
Статья принята:
15.05 .19

\title{
MRI IN THE DIAGNOSIS OF RARE CASE OF PRIMARY MULTIPLE MALIGNANT TUMORS: OSTEOSARCOMA AND UROTHELIAL BLADDER CANCER, PROSTATE ADENOCARCINOMA
}

\author{
Grigorev E.G., Frolova I.G., Perelmuter V.M., Slonimskaya E.M., Usynin E.A., \\ Fesik E.A., Goldberg V.E., Goldberg A.V.
}

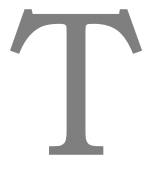

o present the observation of a rare combination of tumor pathology of the bladder, demonstrating the capabilities of magnetic resonance imaging (MRI) in the diagnosis of pelvic tumors.

Materials and methods. Patient G., 68 years old, who complained about the presence of blood in the urine, pain in the abdomen and iliac region. A bladder tumor was suspected. Studies were performed: MRI of the pelvic organs in order to clarify the localization

Cancer Research Institute, Tomsk National Research Medical Center, Russian Academy of Sciences. Tomsk, Russia. 
of education, the degree of invasion and planning for further treatment.

Results. The diagnosis of a bladder tumor is confirmed. Tumors with different histological structure, simulating invasion of the muscular layer of the bladder, are revealed.

Conclusion. The presented case confirms the key role of MRI in the diagnosis of pathology of the pelvic organs, in particular - tumors of the bladder, with the ability to assess the prevalence of the pathological process and treatment planning. However, given the diversity of possible histological variants, pathological confirmation is necessary for the final diagnosis.

Keywords: MRI, dynamic contrast enhancement, DWI, bladder tumor, sarcoma.

Corresponding author: Grigoriev E.G., e-mail: mrtomo@mail.ru

For citation: Grigorev E.G., Frolova I.G., Perelmuter V.M., Slonimskaya E.M., Usynin E.A., Fesik E.A., Goldberg V.E., Goldberg A.V. MRI in the diagnosis of rare case of primary multiple malignant tumors: osteosarcoma and urothelial bladder cancer, prostate adenocarcinoma. REJR 2019; 9(2):288-294. DOI:10.21569/2222-7415-2019-9-2-288-294.
Received:
27.04.19
Accepted:
15.05.19

TH

овообразования мочевого пузыря в подав яющем большинстве случаев имеют эпителиальное происхождение, включая переходноклеточную карциному (более 90\%), плоскоклеточный рак (6-8\%), и аденокарциному (2\%).

Недифференцированный рак встречается менее чем в $1 \%$ случаев $[1,2]$. Злокачественные мезенхимальные опухоли составляют менее 0,04\% змокачественных опухомей мочевого пузыря [3]. Наиболее частым морфологическим вариантом из них явцяется рабдомиосаркома у детей и мейомиосаркома в старшей возрастной группе. В медицинской митературе до настоящего времени удалось найти сообщения только о 32 опубликованных случаях первичной остеосаркомы мочевого пузыря. Из митературы известно о 9 случаях саркоматоидного уротелиального рака мочевого пузыря с элементами остеосаркомы, представменные, однако, единым узцом [4]. Единичны случаи первичномножественных зцокачественных образований, например - остеосаркома мочевого пузыря, одновременно с раком предстательной железы и кишечника [5].

Дия рака мочевого пузыря основным звеном патогенеза считается прямой диительный контакт уротелия с мочой, содержащей экскретируемые канцерогенные вещества $[1,6]$. Этот факт отражается в склонности уротелиального рака к множественному поражению. До 30-35\% больных имеют множественные образования мочевого пузыря [2, 7]. В России выявцение больных с поверхностной формой (1-я стадия) рака составцяет мишь 20-30\%, в то время как в зарубежных странах этот показатель достигает $80 \%$. При этом частота ошибок при опредемении стадии заболевания достигает 73\% [1]. Поэтому своевременная диагностика и мечение больных с опухолями мочевого пузыря остаются актуальными и сложными проблемами современной онкоцогии.

Комплекс методов мучевой диагностики при РМП на сегодняшний день включает, прежде всего, ультразвуковое исследование, рентгенологические методики, компьютерную и магнитно-резонансную томографию $[1,7]$.

Аечебная тактика зависит от степени распространенности процесса, в большинстве случаев показано хирургическое мечение [7].

Клинический случай.

Пациент Г., 68 мет, направцен в клинику НИИ онкологии Томского НИМЦ 10.08.2018 г. дмя прохождения МРТ органов малого таза. Из анамнеза известно: 23.07.2018 г. отметим первый эпизод появцения крови в моче. По месту жительства выполнено УЗИ, при котором на мевой стенке мочевого пузыря определялась опухоль 22х13 мм. Пациент жаловался на боли в подвздошной области при мочеиспускании, отмечено нарастание указанных симптомов, в связи с чем, самостоятельно обратился в НИИ онкологии на консультацию к онкоурологу. В направлении на исследование отмечен предпомагаемый диагноз: "Объемное образование мочевого пузыря", рекомендовано выполнение MPТ. Назначено обследование: общий и биохимический анализы крови, коагулограмма, ЭКГ, компьютерная томография ОГК, УЗИ органов брюшной полости, почек и органов малого таза, остеосцинтиграфия, цистоскопия.

В анализах крови отмечались гиперхоле-

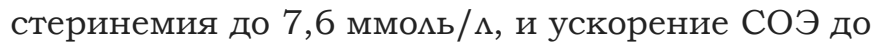
$28 \mathrm{мм} /$; ПСА 2,5 нг/м . При компцексном обследовании данных за отдаленное метастазирование не получено. По данным цистоскопии: в области мевой боковой стенки МП определяется опухоль до 25-30 мм, безворсинчатая, на осно- 


\section{RUSSIAN ELECTRONIC JOURNAL OF RADIOLOGY}

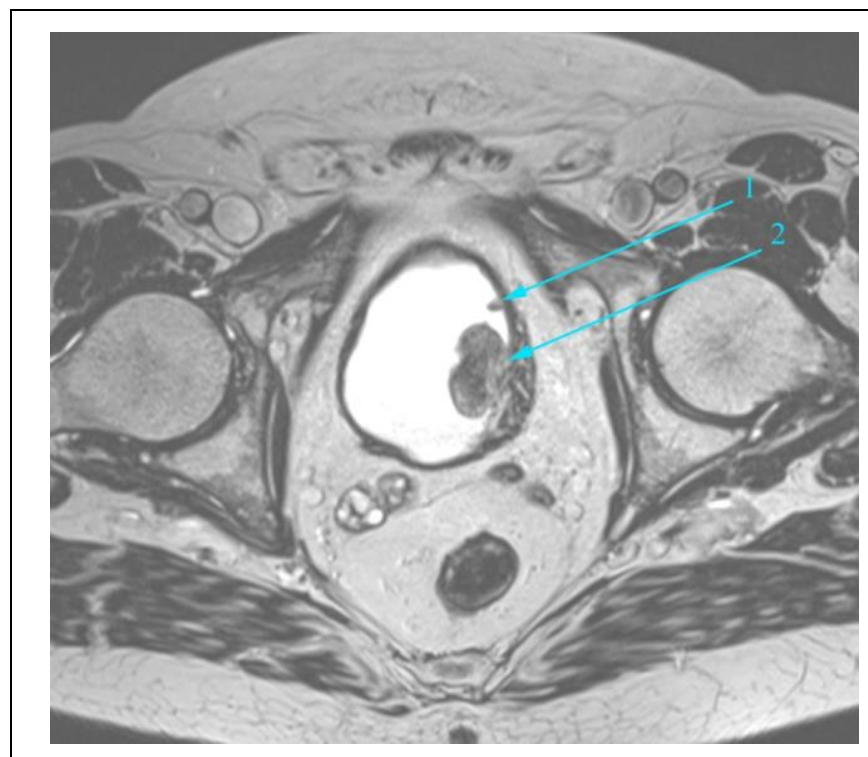

Puc. 1 a (Fig. 1 a)

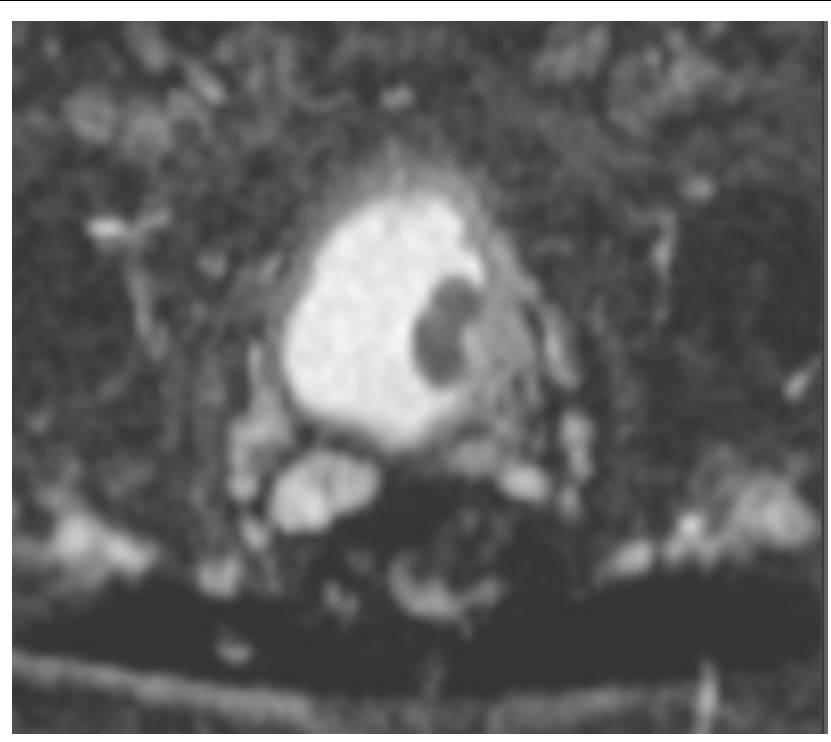

Рис. 16 (Fig. 1 b)

Рис. 1. МРT.

Объёмные образования стенки мочевого пузыря (a-Т2-ВИ, b-ИКД-карта). Гипоинтенсиная уротелиальная опухоль без признаков мышечной инвазии (стрелка 1), более крупная опухоль - остеосаркома с МРпризнаками утолщения и инвазии мышечного слоя (стрелка 2).

Fig. 1. MRI.

Bladder wall tumors (a-T2-WI, b-ADC-DWI), larger - heterogeneous with signs of muscle layer invasion. Hypointense urothelial tumor without signs of muscle invasion (arrow 1), larger tumor - osteosarcoma with MR-signs of thickening and invasion of the muscular layer (arrow 2).

вании среднего диаметра, с участками некроза на поверхности. Выполнен забор биопсии 2 фрагмента.

Пациенту было выполнено МРТ органов малого таза по расширенному протоколу, который применяется при подозрении на опухоль мочевого пузыря. Данный протокоц, дополнительно к стандартным импульсным последовательностям, вкАючал:

- диффузионно-взвешенные изображения co значениями b-фактора 0-50, и 800-900 с/мм2и получением карты измеряемого коэффициента диффузии;

- субмимлиметровое сканирование высокого разрешения с объемом вокселя 0,5-1,0 мм3; (импульсные последовательности T2-Space, Siemens);

- внутривенное динамическое контрастирование гадоминий-содержащим препаратом (болюсно), в дозировке 0,2 мц/кг (0,1 ммоль/кг), со скоростью 1 м / сек с помощью автоматического инжектора.

По данным МР-томографии от 10.08.2018 г.: в боковой стенке мочевого пузыря слева определялось объемное образование (рис. 1), деформирующее просветный контур, мокализовалось кпереди от устья мочеточника на 3-5 часах, на широком основании ( 18-19 мм). Образование мягкотканое, однородной структуры, с относительно четкими, бугристыми просветными контурами, размеры составляли до 30х18х2 1 мм (переднезадний $\mathrm{x}$ поперечный х вертикальный). Устье мочеточника без видимой деформации. Отмечалось неравномерно выраженное утолщение стенки пузыря в основании опухоми, до 5-8,5 мм, структура стенки была неоднородной, в большей степени за счет обогащения сосудистого рисунка (рис. 1).

При внутривенном контрастировании отмечалось интенсивное однородное накопмение парамагнетика выявценным образованием, по контуру слизистой и глубже, непосредственно в мышечном слое стенки мочевого пузыря (рис. 2). Перивезикальная кцетчатка достаточно однородна, структурна.

Отмечались более мемкие образования у шейки мочевого пузыря - до 4 мм, и в боковой стенке слева, до 5,5 мм (рис. 1, 2).

Предстательная железа асимметрично увеличена, с признаками умеренной жемезистостромальной гиперплазии переходных зон. Периферическая зона с участками фиброза и отёка, более выраженного справа. Очаговых изменений не отмечено. При внутривенном контрастировании асимметричного очагового раннего усиления не выявцено. Усицена васкуляризация перипростатического венозного сплетения.

Заключение: Объёмные образования мо- 


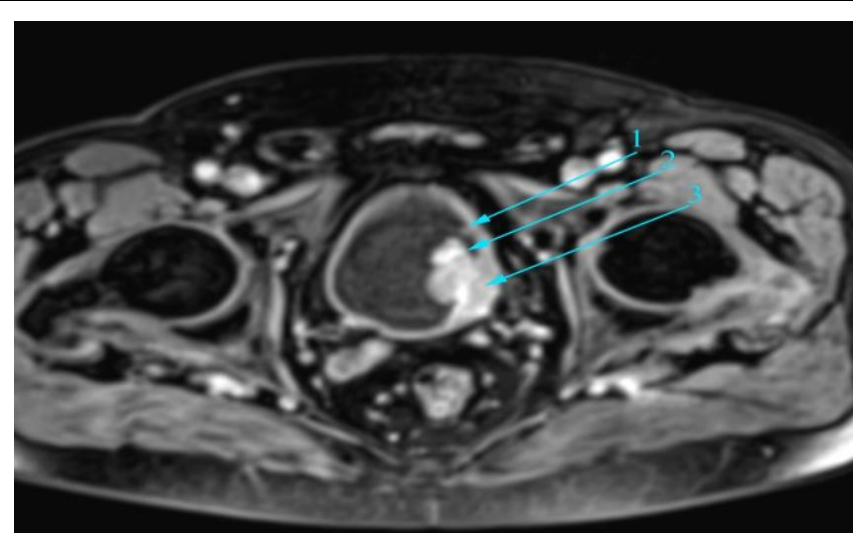

Pис. 2 (Fig. 2)

\section{Рис. 2. МРТ (T1-fatsat) с внутривенным контра- стированием.}

Слабоинтенсивное накопление парамагнетика опухолью уротелия (стрелка 1), неоднородное интенсивное накопление парамагнетика в структуре более крупного образования (стрелка 2), и подобное накопление контраста в утолщенном мышечном слое стенки пузыря (стрелка 3).

\section{Fig. 2. MRI (T1-fatsat) with intravenous contrast.}

Low-intensity accumulation of contrast by the urothelium tumor (arrow 1), non-uniform intensive accumulation of the paramagnetic in the structure of a larger formation (arrow 2), and similar accumulation of contrast in the thickened muscle layer of the bladder wall (arrow 3).

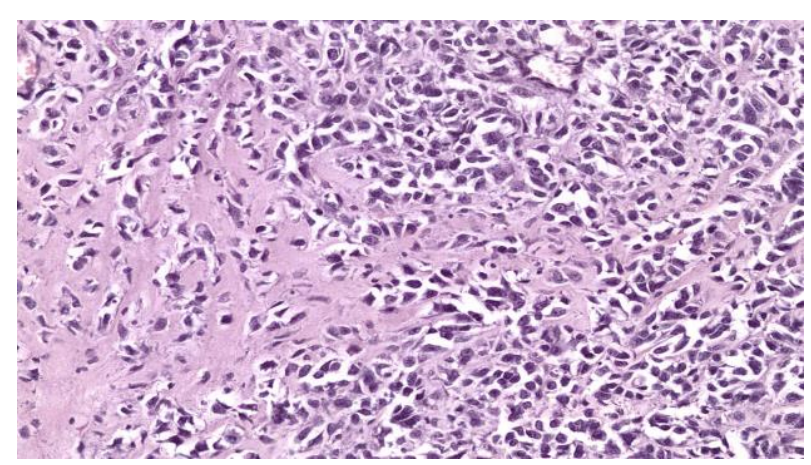

Puc. 4 a (Fig. 4 a)

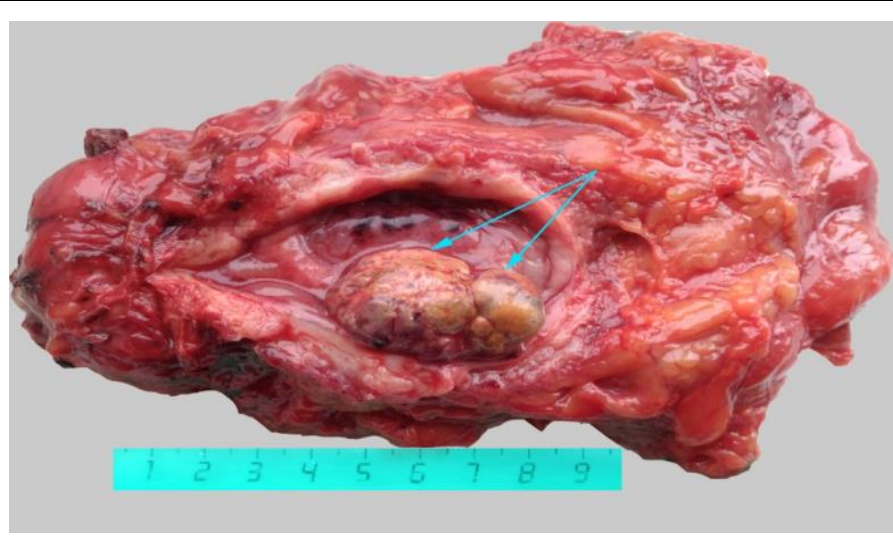

Pис. 3 (Fig. 3)

\section{Рис. 3. Макропрепарат.}

Фрагмент удаленного мочевого пузыря с клетчаткой. Опухоль желто-серого цвета (стрелка), деформирующая просвет мочевого пузыря.

\section{Fig. 3. Gross specimen.}

Fragment of the removed bladder with fat tissue. The tumor is yellow-gray, deforming the bladder lumen.

\section{Рис. 4. Остеосаркома мочевого пузыря, G3.}

а - Окраска гематоксилин-эозин, ув.х100. Опухоль представлена крупными умеренно полиморфными клетками с нормохромным ядром, с неширокой эозинофильной цитоплазмой. Опухолевые клетки межат в атипическом остеоиде.

б - Иммуногистохимическое исследование, ув.х100. В опухолевых клетках отмечается яркая ядерная экспрессия SATB-2 (clone EP281, Ctll Marque).

\section{Fig. 4. Osteosarcoma of the bladder, G3.}

a - Hematoxylin-eosin, x100. The tumor was constructed by large, moderately polymorphic cells with a normochromic nucleus, with a narrow eosinophilic cytoplasm. Tumor cells lie in the atypical osteoid.

b - Immunohistochemical study, x100. Bright nuclear expression of SATB-2 (clone EP281, Ctll Marque) is observed in tumor cells. 
чевого пузыря, более крупное слева - с признаками инвазии мышечного слоя. Признаки ДГПЖ, простатита.

В целом, по МРТ не предполагалось конкретная гистологическая форма опухоми мочевого пузыря, но по МР-характеристикам отмечено отличие в классической МР-картине и семиотике данного образования от более часто встречающегося уротелиального рака. В частности, при таких размерах, контуры опухоми не соответствовали "классическому" варианту по типу "цветной капусты", также отмечалось утолщение подмежащей стенки с обогащением сосудистого рисунка, и неоднородное накопцение контрастного препарата в утолщенном мышечном слое стенки пузыря (рис. 2). В предстательной железе признаков узцовых образований не выявлено.

На этапах обследования жалобы и симптомы сохранялись прежней степени выраженности. Пациенту было показано оперативное мечение. 22.08.2018 г. выпомнена радикальная цистпростатэктомия, биматеральная мимфаденэктомия, гетеротопическая тонкокишечная пластика фрагментом подвздошной кишки. Швы сняты на 14-е сутки. Заживление первичным натяжением.

По данным гистологического исследования № 23308-32/ 18 от 30.08.18 г.:

Получено заключение: Синхронные первично-множественные зцокачественные образования (ПМЗО): остеосаркома мочевого пузыря без инвазии в мышечный слой (рис. 4), уротелиальная карцинома in situ (рис. 5), ацинарная аденокарцинома предстательной железы с поражением обеих домей $6(3+3)$ по ГАиссону, без поражения мимфатических узмов и семенных пузырьков (рис. 6).

Границы резекции без опухомевой ткани. Аимфатические узцы без признаков метастатического поражения.

Катамнез: Выполнены контрольные анамизы мочи и крови, УЗИ малого таза. Пациент бым выписан под наблюдение онколога по месту жительства, с контролем ПСА, МРТ малого таза, УЗИ почек, рентгенографии мегких раз в 6 месяцев.

\section{Обсуждение.}

Остеосаркома мочевого пузыря является редкой патологией. В большинстве случаев опухоли, поражающие стенку мочевого пузыря представцены вариантами уротелиальной карциномы. Манифестируют и диагностируются при обследовании пациентов в связи с гематурией. У большинства пациентов диагноз подтверждается при использовании УЗИ, цистоскопии. Магнитно-резонансная томография обеспечивает наиболее высокую, среди остальных модамьностей, тканевую контрастность, позволяет выявить количество, размеры, мокамизацию образований мочевого пузыря, их структурные характеристики, состояние перивезикальной клетчатки и окружающих органов и мимфатических узцов. Считается, что точность МРТ в стадировании первичной опухоли варьирует от 73 до 96 \% [8]. При контрастировании на динамических сканах опухоль демонстрирует более интенсивное повышение

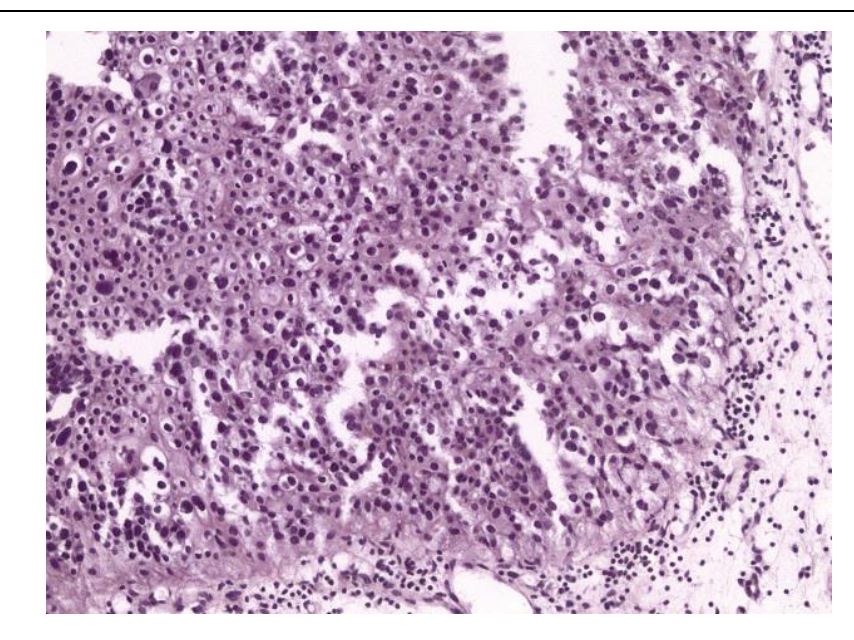

Рис. 5 (Fig. 5)

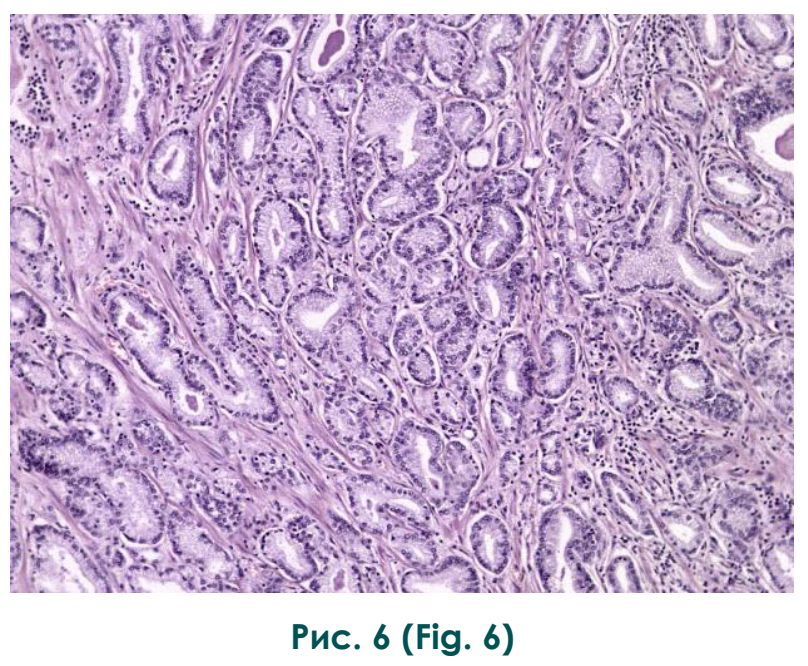

Рис. 6. Микропрепарат.

Рис. 5. Микропрепарат.

Уротелиальная carcinoma in situ. Окраска гематоксилин-эозин, ув.х 100.

Fig. 5. Micropreparation.

Urothelial carcinoma in situ. Hematoxylin-eosin, x100.
Ацинарная аденокарцинома предстательной железы, 6 балов по ГАисону. Окраска гематоксилин-эозин, ув.х100.

\section{Fig. 6. Micropreparation.}

Acinar adenocarcinoma of the prostate, Gleason 6. Hematoxylin-eosin, x100. 
МР-сигнала, чем нормальная стенка мочевого пузыря ими постбиопсийные изменения [9]. Мышечный слой стенки мочевого пузыря домьше, чем ткань опухоли, остается гипоинтенсивным на Т1-ВИ, что позволяет более четко дифференцировать слизистый и мышечный слои, и, соответственно, с большой точностью опредемять распространение патологического процесса в данных слоях стенки мочевого пузыря [8].

На сегодняшний день при градации вероятности инвазии опухоли в стенку мочевого пузыря предможены критерии критерии VI-RADS (Vesical Imaging-Reporting And Data System) при проведении мультипараметрической МРТ [10]. Однако в представленном наблюдении накопмение парамагнетика в утолщенной стенке отмечалось с такой же интенсивностью, как в просветном компоненте образования. При этом, по данным ИКД-карты диффузионновзвешенных изображений отмечается ограничение диффузии только просветным компонентом опухоли (рис. 1b). Таким образом, данные Т2-взвешенных изображений и динамического контрастирования соответствовали VI-RADS 5 (высокая вероятность мышечной инвазии), тогда как диффузионно-взвешенные изображе-

\section{Cписок питературы:}

1. Григорьев Е.Г., Фролова И.Г., Усынин Е.А., Величко С.А., Окунев В.В., Гольдберг А.В. Рак мочевого пузыря: возможности высокопольной магнитно-резонансной томографии (Обзор литературы). Сибирский онкологический журнал. 2014; 3: 65-71.

2. Epstein JI, Amin M, Reuter VE. Biopsy interpretation of the bladder. Wolters Kluwer Health Adis (ESP), 2012. 355 p.

3. Фролова И.Г., Григорьев Е.Г., Трухачева Н.Г., Усова А.В. Возможности высокоразрешаюших протоколов магнитнорезонансной томографии в диагностике рака мочевого пузыря. Медииинская визуализаиия. 2016; 2 (1): 49-56.

4. Tanuma, K., Kawai, K., Tsuchiya, H., Matsumoto, Y., Kandori, S., Kojima, T., Sakata, A. Sarcomatoid Urothelial Carcinoma of the Bladder Including an Osteosarcoma Element. Hinyokika kiyo. Acta urologica Japonica. 2017; 63 (11): 487-492. DOI: 10.14989/ActaUrolJap_63_11_487.

5. Ghalayini I. F., Bani-Hani I. H., Almasri N. M. Osteosarcoma of the urinary bladder occurring simultaneously with prostate and bowel carcinomas: report of a case and review of the literature. Archives of pathology \& laboratory medicine. 2001; 125

\section{References:}

1. Grigoriev, E.G., Frolova, I.G., Usynin, E.A., Velichko, S.A., Okunev, V.V., Goldberg, A.V. Bladder cancer: the possibilities of high-field magnetic resonance imaging (Literature review). Siberian Oncology Journal. 2014; 3: 65-71 (in Russian).

2. Epstein JI, Amin M, Reuter VE. Biopsy interpretation of the bladder. Wolters Kluwer Health Adis (ESP), 2012. 355 p.

3. Frolova I.G., Grigoriev E.G., Trukhacheva N.G., Usova A.V. Possibilities of high-resolution magnetic resonance imaging protocols in the diagnosis of bladder cancer. Medical imaging.2016; ния и ИКД-карта соответствовали VI-RADS 2 (низкая вероятность мышечной инвазии). Выявменные семиотические данные, вероятно, отмичают представценный вариант мезенхимальной опухоли от более часто встречающейся уротелиальной карциномы, однако подтвердить этот тезис пока невозможно вследствие редко встречающейся патологии.

Также особенностью представценного случая является отсутствие признаков опухоцевой патологии предстательной железы по данным МРТ, что в случае изолированного поражения (без проведенного мечения) могмо привести к прогрессированию заболевания. Плановое гистологическое исследование операционного материала позволимо более точно оценить распространенность опухолевого процесса и поставить окончательный диагноз.

Источник финансирования и конфликт интересов.

Авторы данной статьи подтвердими отсутствие финансовой поддержки исследования и конфиикта интересов, о которых необходимо сообщить.

\section{(6): $\quad 793-795 . \quad$ DOI: $\quad 10.1043 / 0003$}

9985(2001)125<0793:OOTUBO>2.0.CO;2

6. Коробкин А.С., Терновой С.К., Пушкарь Д.Ю. Комплексная диагностика рака мочевого пузыря. REJR. 2012; 2 (4): 74-78. 7. Down CJ, Nair R, Thurairaja R. Bladder cancer. Surgery (Oxford). 2016; 34 (10): 532-9. DOI: 10.1016/j.mpsur.2016.08.001

8. Alfred Witjes J, Lebret T, Compérat EM, Cowan NC, De Santis M, Bruins HM, et al. Updated 2016 EAU Guidelines on Muscleinvasive and Metastatic Bladder Cancer. European Urology. $\begin{array}{lllll}\text { Elsevier BV. 2017; } 71 & \text { (3): 462-75. DOI: }\end{array}$ 10.1016/j.eururo.2016.06.020

9. Babjuk M, Böhle A, Burger M, Capoun O, Cohen D, Compérat EM, et al. EAU Guidelines on Non-Muscle-invasive Urothelial Carcinoma of the Bladder: Update 2016. European Urology. $\begin{array}{lllll}\text { Elsevier BV. 2017; } 71 & \text { (3): 447-61. DOI: }\end{array}$ 10.1016/j.eururo.2016.05.041.

10. Panebianco V, et al. Multiparametric Magnetic Resonance Imaging for Bladder Cancer: Development of VI-RADS (Vesical Imaging-Reporting And Data System). Eur Urol. 2018. DOI: 10.1016/j.eururo.2018.04.029.
2 (1): 49-56 (in Russian).
4. Tanuma, K., Kawai, K., Tsuchiya, H., Matsumoto, Y., Kandori,
S., Kojima, T., Sakata, A. Sarcomatoid Urothelial Carcinoma of the Bladder Including an Osteosarcoma Element. Hinyokika kiyo. Acta urologica Japonica. 2017; 63 (11): 487-492. DOI: 10.14989/ActaUrolJap_63_11_487.
5. Ghalayini I. F., Bani-Hani I. H., Almasri N. M. Osteosarcoma of the urinary bladder occurring simultaneously with prostate and bowel carcinomas: report of a case and review of the litera- 


\section{RUSSIAN ELECTRONIC JOURNAL OF RADIOLOGY}

ture. Archives of pathology \& laboratory medicine. 2001; 125 (6): $\quad 793-795 . \quad$ DOI: $10.1043 / 0003$ 9985(2001)125<0793:OOTUBO>2.0.CO;2

6. Korobkin A.S., Ternovoy S.K., Pushkar D.Yu. Comprehensive diagnosis of bladder cancer. REJR. 2012; 2 (4): 74-78 (in Russian).

7. Down CJ, Nair R, Thurairaja R. Bladder cancer. Surgery (Oxford). 2016; 34 (10): 532-9. DOI: 10.1016/j.mpsur.2016.08.001 8. Alfred Witjes J, Lebret T, Compérat EM, Cowan NC, De Santis M, Bruins HM, et al. Updated 2016 EAU Guidelines on Muscleinvasive and Metastatic Bladder Cancer. European Urology. $\begin{array}{llllll}\text { Elsevier } \quad \text { BV. 2017; } 71 & \text { (3): } 462-75 . \quad \text { DOI: }\end{array}$ 10.1016/j.eururo.2016.06.020

9. Babjuk M, Böhle A, Burger M, Capoun O, Cohen D, Compérat $E M$, et al. EAU Guidelines on Non-Muscle-invasive Urothelial Carcinoma of the Bladder: Update 2016. European Urology. Elsevier BV. 2017; 71 (3): 447-61. DOI: 10.1016/j.eururo.2016.05.041.

10. Panebianco $V$, et al. Multiparametric Magnetic Resonance Imaging for Bladder Cancer: Development of VI-RADS (Vesical Imaging-Reporting And Data System). Eur Urol. 2018. DOI: 10.1016/j.eururo.2018.04.029. 